\title{
Quantum and classical ripples in graphene
}

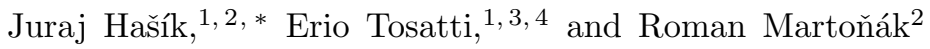 \\ ${ }^{1}$ International School for Advanced Studies (SISSA), Via Bonomea 265, I-34136 Trieste, Italy \\ ${ }^{2}$ Department of Experimental Physics, Comenius University, Mlynská Dolina F2, 842 48 Bratislava, Slovakia \\ ${ }^{3}$ CNR-IOM Democritos, Via Bonomea 265, I-34136 Trieste, Italy \\ ${ }^{4}$ The Abdus Salam International Centre for Theoretical Physics (ICTP), Strada Costiera 11, I-34151 Trieste, Italy
}

(Dated: April 12, 2018)

\begin{abstract}
Thermal ripples of graphene are well understood at room temperature, but their quantum counterparts at low temperatures are in need of a realistic quantitative description. Here we present atomistic path-integral Monte Carlo simulations of freestanding graphene, which show upon cooling a striking classical-quantum evolution of height and angular fluctuations. The crossover takes place at ever-decreasing temperatures for ever-increasing wavelengths so that a completely quantum regime is never attained. Zero-temperature quantum graphene is flatter and smoother than classical graphene at large scales, yet rougher at short scales. The angular fluctuation distribution of the normals can be quantitatively described by coexistence of two Gaussians, one classical strongly T-dependent and one quantum about $2^{\circ}$ wide, of zero-point character. The quantum evolution of ripple-induced height and angular spread should be observable in electron diffraction in graphene and other two-dimensional materials, such as $\mathrm{MoS}_{2}$, bilayer graphene, boron nitride, etc.
\end{abstract}

Suspended graphene is a unique physical realization of a polymerized two-dimensional (2D) membrane embedded in three-dimensional space. Its equilibrium behavior, characterized by the large distance limit $\Lambda$ of angular correlations $\langle\vec{n}(\overrightarrow{0}) \cdot \vec{n}(\vec{x})\rangle \rightarrow \Lambda$ of tangent plane normal vectors $\vec{n}(\vec{x})$, is conventionally termed crumpled if $\Lambda$ vanishes, flat if not 1, 2]. Like other membranes, graphene is endowed with ultra-soft out-of-plane "flexural" acoustic modes of dispersion $\omega=\sqrt{\frac{\kappa}{\rho}} q^{2}$, where $\kappa$ is the bending rigidity, $\rho$ is the area mass density, and $q$ is the wave vector. In the classical harmonic approximation, as a consequence of equipartition, each such mode acquires an average energy $k_{B} T$, leading to a height-height correlation function decaying in the long-wavelength limit as $\left\langle\left|h_{q}\right|^{2}\right\rangle \sim q^{-4}$. That would lead to growth of height meansquare fluctuations $\left\langle[h(\vec{x})-h(0)]^{2}\right\rangle^{1 / 2} \sim|\vec{x}|$ destabilizing the flat phase at any finite temperature. In reality that does not happen because of the long-recognized anharmonic coupling of the flexural modes to the more regular stiffer in-plane acoustic modes 3 , a coupling reflecting the physical necessity for the membrane's planar extension to shrink when corrugated. Its effect on the classical thermal equilibrium state of the membrane was studied by renormalization-group (RG) methods [2 [5] and by classical Monte Carlo (MC) [6 8] and molecular dynamics (MD) simulations 9] leading, despite some variants [10, to a consistent picture. Anharmonic coupling increases the $q \rightarrow 0$ bending rigidity from the constant $\kappa$ to $q$-dependent $\kappa(q) \sim q^{-\eta}$ which stiffens the membrane and renormalizes the long-wavelength height fluctuations $\left\langle\left|h_{q}\right|^{2}\right\rangle$ from $\left\langle\left|h_{q}\right|^{2}\right\rangle \sim q^{-4}$ to $\left\langle\left|h_{q}\right|^{2}\right\rangle \sim q^{-4+\eta}$. With $\eta \sim 0.8-0.85$, the state of the membrane is restored from crumpled to flat 2 .

\footnotetext{
*jhasik@sissa.it
}

So far we discussed the known classical picture. The low-temperature quantum membrane must behave differently. Already at the harmonic level the $\omega$-independent equipartition energy $k_{B} T$ per mode is replaced by the zero-point energy $\frac{1}{2} \hbar \omega$. That yields the very different height-height correlation function $\left\langle\left|h_{q}\right|^{2}\right\rangle \sim q^{-2}$ (Ref.[4]), representing a flat quantum ground state as opposed to the crumpled classical harmonic state at finite temperature 11]. Compared to thermal fluctuations, quantum zero-point motion implies weaker longwavelength flexural fluctuations, yet relatively enhanced short-wavelength ones, suggesting that quantum ripples will make graphene globally flatter but locally rougher than their classical counterpart. The actual rippling of freestanding graphene including quantum fluctuations and anharmonic interaction between the modes was addressed in a series of recent theoretical approaches [5, 1113, whose predictions are limited to the long-wavelength behaviour of correlations. A complementary study of quantum and anharmonic effects on the flexular mode was performed in Ref. 14 by lattice dynamical methods. Path-integral Monte Carlo (PIMC) 15] and path-integral molecular dynamics simulations [16-18] addressed at the atomistic level some important thermodynamic quantities. We address here the quantities of crucial physical interest, the height-height correlation function and the angular distribution of surface normals.

Here we present extensive PIMC atomistic simulations, whose results, besides agreeing with RG asymptotics, provide also a fully realistic description of height correlations and angular profiles of graphene under quantum and thermal fluctuations, whose evolution from classical to quantum is revealing in view of ongoing experimental tests.

Graphene was modeled by a fully mobile honeycomb lattice of $N$ pointlike atoms (initially $N=19440$, 


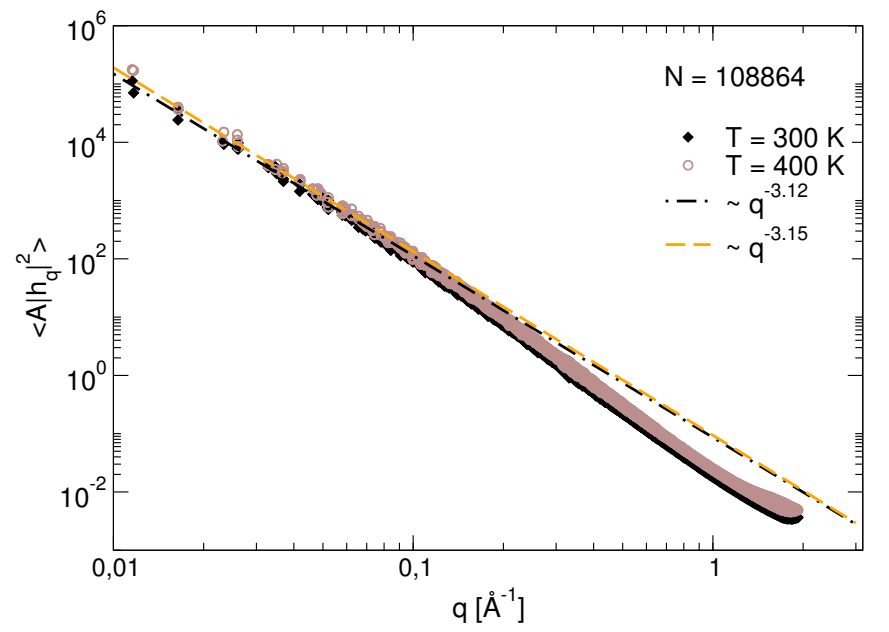

FIG. 1. Classical graphene height-height correlation function for $N=108$ 864. The long-wavelength asymptotic slope $-4+$ $\eta$ equals $-3.12 \pm 0.05$ at $300 \mathrm{~K}$ and $-3.15 \pm 0.05$ at $400 \mathrm{~K}$, $\eta$ reflecting the effect of anharmonic fluctuations. This can be compared to the classical behaviour for the smaller system $N=4860$ at $T=0.6 \mathrm{~K}$ shown in Fig. 3 where the slope is -3.98 , as expected for purely harmonic fluctuations.

scaling down to 4860 for quantum PIMC at very low temperatures, and up to 108864 for classical MC) interacting through Tersoff's empirical potential [19, 20]. This potential was optimized to reproduce experimental phonon-dispersion relations of in-plane graphite and is computationally less demanding than the reactive bondorder potential 21] used in the series of MC simulations [6] 8]. The single 2D membrane is replaced in PIMC by imaginary-time-coupled copies (Trotter slices), whose number $M$ must be large enough at each given temperature $T$ to warrant convergence. Details about simulations, the PIMC method [22 25], and Trotter convergence are further discussed in the Supplemental Material (SM) 26].

As an initial test of the MC simulation method we first calculated the height-height correlation function in the classical case at $T=300$ and $400 \mathrm{~K}$. The results for $N=108864$ (linear size $530 \AA$ ) are shown in Fig. 1 (in this and all subsequent figures the height-height correlation function is normalized by the system area $A$, see Computational Methods, SM). The long-wavelength height fluctuations show two distinct regimes, crossing over at $q \sim 0.1 \AA^{-1}$ from harmonic $q^{-4}$ to anharmonic $q^{-4+\eta}$ with $\eta=0.85-0.88$, in good agreement with RG predictions, and with numerical simulations 2, 8, 11] claims that the classical correlation function should behave for $q \rightarrow 0$ as $\left\langle\left|h_{q}\right|^{2}\right\rangle \sim q^{-2}$ [10] are therefore not substantiated.

Satisfied by this check we moved on to main objective of our work - quantum PIMC simulations. In anticipation of increasing system size along the Trotter dimension, we start with a smaller membrane of $N=19440$ atoms and

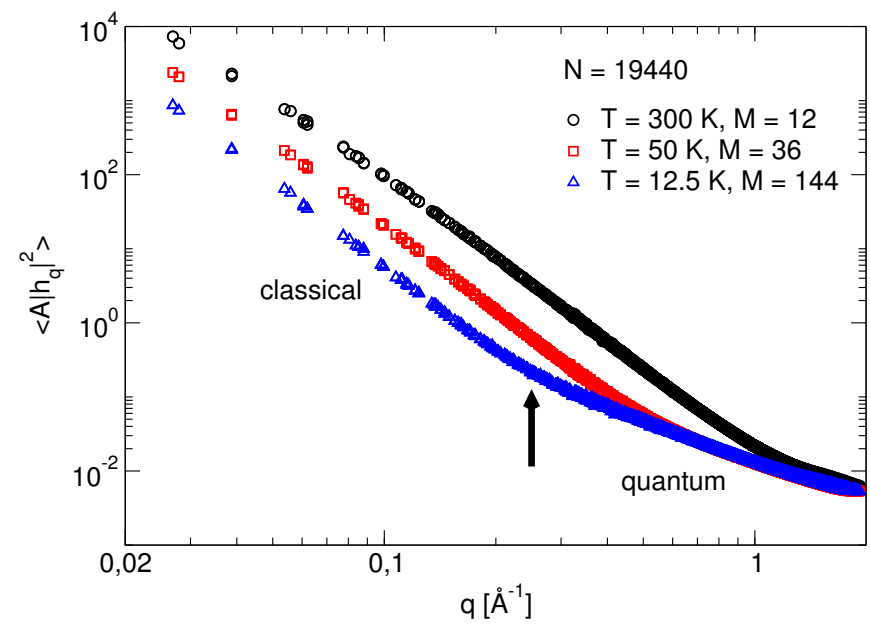

FIG. 2. Graphene PIMC height correlations for $N=19440$. The arrow marks the crossover from thermal to quantum regime for $T=12.5 \mathrm{~K}$. The classical regime is visible at small $q$, whereas the quantum regime emerges at large $q$.

proceed by cooling it down. Figure 2 shows the PIMC height correlation function for $N=19440(220 \AA$ linear size) at $T=300,50$ and $12.5 \mathrm{~K}$. Both $T=50$ and $12.5 \mathrm{~K}$ data show a clear crossover from classical correlations for $q<q_{T}$ to quantum ones taking place, again in agreement with predictions, for $q>q_{T}$, where [11, 27.

$$
q_{T}=\left(\sqrt{\frac{\rho}{\kappa}} \frac{2 k_{B} T}{\hbar}\right)^{1 / 2}
$$

marks the point where the classical equipartition energy of the flexural phonon equals its quantum zero-point en-

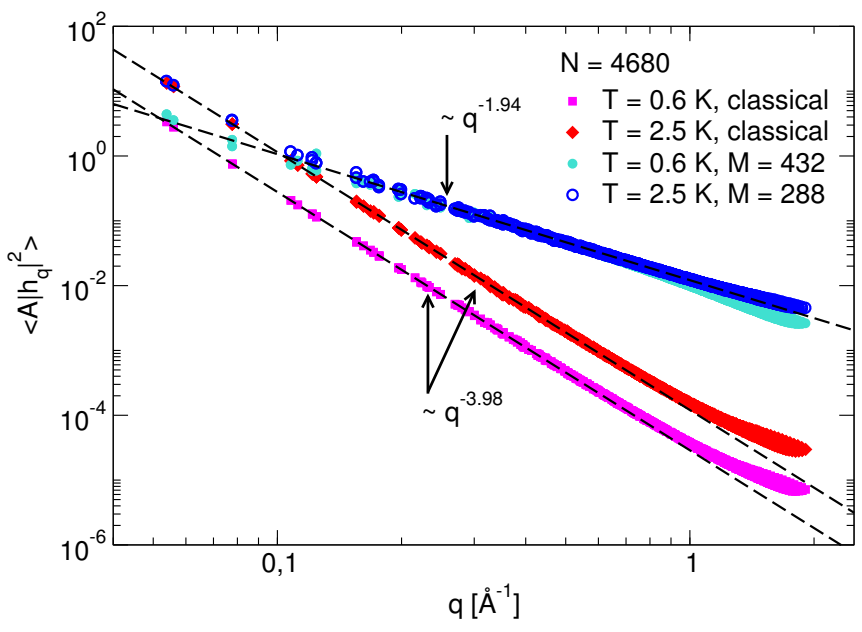

FIG. 3. Height-height correlations for $N=4860$ at $T=0.6$ and $T=2.5 \mathrm{~K}$ : classical and PIMC ( $M=288$ and $M=432)$. The region $q<0.6 \AA^{-1}$ is fully converged with respect to the Trotter number $M$. The slope of the classical curves is $-3.98 \pm 0.003$ while that of the quantum curves is $-1.94 \pm 0.02$ for $q>q_{T}$. 


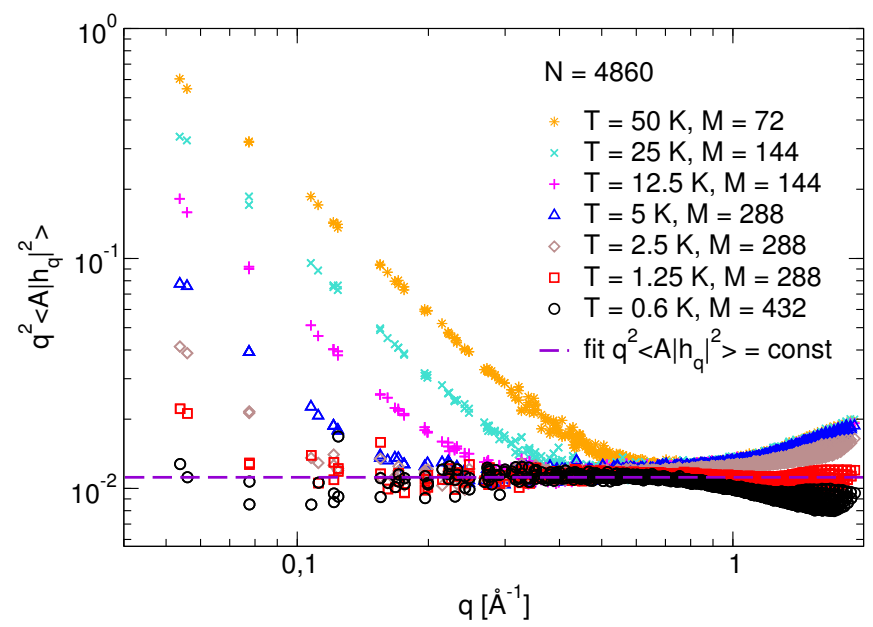

FIG. 4. Temperature-dependent $N=4860$ PIMC height correlations of graphene, multiplied by $q^{2}$ so that $q^{-2}$ behaviour appears as horizontal. The gradual temperature-dependent veering from quantum correlations towards classical ones at longer wavelengths is clearly visible. The violet horizontal dashed line represents the best fit of $T=0.6 \mathrm{~K}$ data in the region $q<0.6 \AA$ to the quantum harmonic approximation prediction $q^{2} A\left\langle\left|h_{q}\right|^{2}\right\rangle=$ const and serves as a guide to the eye.

ergy, $\frac{1}{2} \hbar \omega \approx k_{B} T$. Curves at $T=50$ and $12.5 \mathrm{~K}$ collapse for $q>0.5 \AA^{-1}$ but diverge for lower $q$-vectors. The quantum result representing the $T=0$ correlations is gradually emerging as the lower envelope of finitetemperature curves at large $q$. For this relatively large system, however, it is hard to push temperature down any further. At $T=12.5 \mathrm{~K}$ the total number of simulated particles is $144 \times 19440 \approx 3 \times 10^{6}$.

By halving the linear size to $L=110 \AA$ with $N=4860$ (lowest- $q$-value $q_{\min }=2 \pi / L \sim 0.057 \AA^{-1}$ ), we could reach much lower temperatures down to $T=0.6 \mathrm{~K}$. The resulting classical and PIMC height-height correlations at $T=2.5$ and $T=0.6 \mathrm{~K}$ are shown in Fig. 3 the first striking result of this paper. It can be seen that the PIMC curves at both temperatures coincide (apart from region $q>0.6 \AA^{-1}$ where the Trotter convergence is not perfect) down to $q \sim 0.1 \AA^{-1}$ where at $T=2.5 \mathrm{~K}$ the classical behaviour takes over. On the other hand, the PIMC curve at $T=0.6 \mathrm{~K}$ shows the quantum regime in the whole region of $q$-vectors spanning about one decade. The PIMC and classical curves are very different, the quantum slope very close to the harmonic value of 2 , compared to about 4 of the classical case. The PIMC height correlations for all simulated temperatures (now multiplied by $q^{2}$ for convenience) are shown in Fig. 4 Ignoring points above $q \approx 0.6 \AA^{-1}$, whose scatter is caused by incomplete Trotter convergence, two regimes are again visible, large $q$ and small $q$. The first shows a flat horizontal behaviour, similar to that expected in the quantum harmonic case, $q^{2}\left\langle\left|h_{q}\right|^{2}\right\rangle \sim$ const. The second, for low $q$, represents the
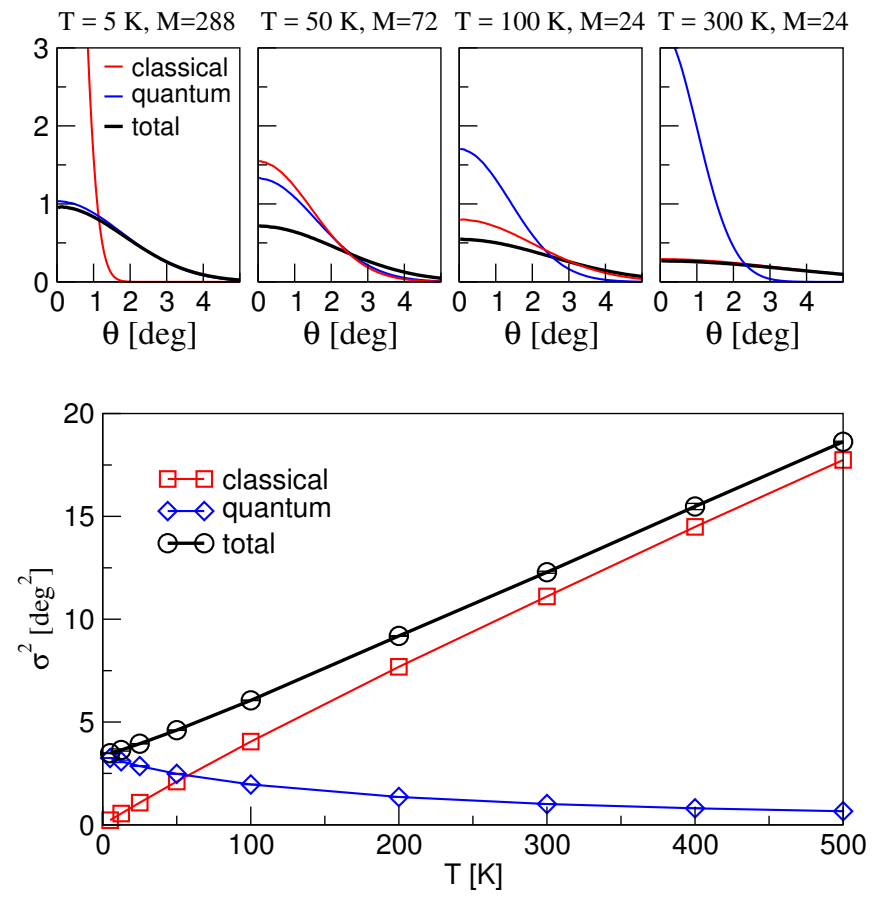

FIG. 5. (Top) Angular probability distribution of graphene's normal for $N=4860$, decomposed into classical and quantum contribution for increasing temperatures. (Bottom) Temperature dependence of the total variance $\sigma^{2}$ of the distribution of normal angles and its classical and quantum components. The variance was determined by fitting the curves to the Gaussian form $C \exp \left(-\frac{\theta^{2}}{2 \sigma^{2}}\right)$.

classical regime. The crossover between the two is again at $q=q_{T}$. At the lowest temperature of $0.6 \mathrm{~K}$ the quantum behaviour therefore extends down to the smallest $q$-vector $q \approx 0.057 \AA^{-1}$. One can conclude that at any given temperature $T$ the ripples of graphene are quantum and of moderate amplitude for shorter wavelengths than $\lambda_{T}=2 \pi / q_{T} \sim T^{-(1 / 2)}$, stronger and classical for longer wavelengths, but never disappearing at any finite temperatures. The temperature dependence of the crossover $q_{T}$ extracted from Fig. 4 is shown in the SM, Fig. 4. Our results suggest, in agreement with Ref. [1] that quantum fluctuations leave the membrane flat on the large scale. The interesting logarithmic corrections implied by $\mathrm{D}=$ 2 being the upper critical dimension of quantum membranes [11] cannot be addressed here because of computational limitations. Accessing even lower $q$ requires both larger system size and lower temperatures, which in turn imply higher Trotter number, and such simulations appear at the moment prohibitively expensive.

A complementary feature and consequence of graphene's ripples, accessible to measurement together with height correlations, is provided by the angular distribution $P(\theta)$ of $\theta$, defined for each atom as the angle between the normal to the triangle formed by its three neighbours and the normal to the averaged membrane 


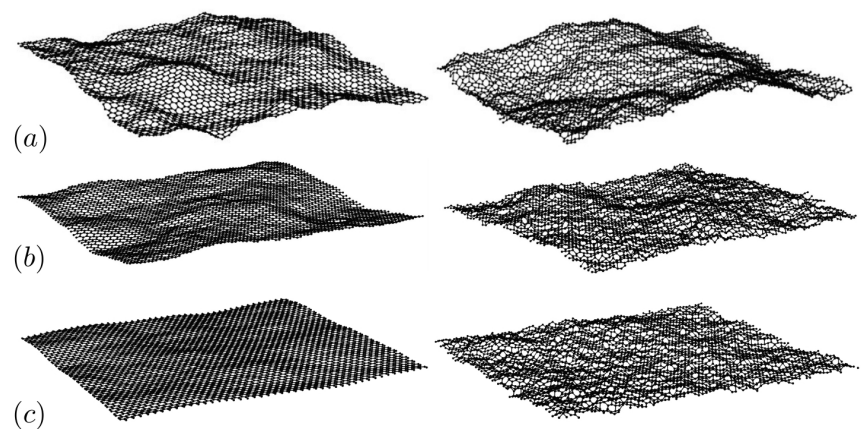

FIG. 6. Comparison of configurations from classical simulations (left) with a single Trotter slice from the PIMC with M Trotter slices (right) for $N=4860$ at different temperatures. (a) $T=50 \mathrm{~K}, M=72$; (b) $T=12.5 \mathrm{~K}, M=144$; (c) $T=2.5$ $\mathrm{K}, M=288$. For convenience, out-of-plane displacements are amplified by a factor 10 .

plane. On this atomistic scale, all fluctuations including those at large $q$ simultaneously contribute to the angular spread. Classically, $P(\theta)$ should evolve from $\delta(\theta)$ at $T=0$ to a Gaussian of finite width at $T>0$. Whereas PIMC simulations fully account for both classical and quantum contributions to the angular spread, they also allow very naturally to decompose the total averaged fluctuations in the two distinct contributions. Generally, the classical contribution is given by the fluctuations of the centroids of Trotter beads averaged over all imaginary time slices whereas the quantum contribution is represented by the fluctuations around the centroids within the slice. In our case we calculated first the classical normal angle distribution for the configuration given by centroids; the angle between the normal in each Trotter slice and the classical one represents the quantum contribution. The analysis of the probability distribution $P(\theta)$ represents the second important result of this paper. The distributions of total, classical, and quantum normal angle fluctuations calculated at several temperatures from 5 to $300 \mathrm{~K}$ are shown in Fig. 5 (upper panel). All of them are rather accurately Gaussian, with a $T$-dependent width or spread $\sigma$. At $T=5 \mathrm{~K}$ the total spread is dominated by the quantum contribution; at $50 \mathrm{~K}$ classical and quantum compete; and at $300 \mathrm{~K}$ the classical prevails over the (declining) quantum.

The crossover between classical and quantum behaviour for normal angle fluctuations is thus around 50 $\mathrm{K}$, slightly lower than expected for some other thermodynamic quantities [5]. Figure 5 (lower panel) shows the temperature dependence of the spread of all distributions. Whereas at room temperature the quantum spread is much smaller than the classical one, at $T=50 \mathrm{~K}$ the quantum one takes over upon cooling, saturating at very low temperatures at a value of $\sigma$ of roughly $2^{\circ}$. The histogram of normal angles at $T=5 \mathrm{~K}$ including the $\sin \theta$ factor from the solid angle element (see the SM,
Fig. 5) shows that the normal angle fluctuations reach up to $4^{\circ}$ and the most likely value of the angle $\theta$ is around $\sigma=1.8^{\circ}$. This substantial residual angular spread represents a rather spectacular manifestation of quantum zero-point motion in a macroscopic membrane.

A pictorial idea of the difference between classical and quantum graphene at different temperatures is offered by simulation snapshots for a variety of cases (Fig. 6) of the $N=4860$ membrane. In the bottom panel (c) we see that at $T=2.5 \mathrm{~K}$ the classical membrane would be nearly flat with just small long-wavelength ripples. Quantum graphene, which at that temperature is close to its ground state, sports instead sizable short-wavelength ripples, which make it locally much rougher. This roughness is also responsible for the broader angular distribution of the normals shown in Fig. 5. In the middle [(b), $T=12.5 \mathrm{~K}]$ and top $[(\mathrm{a}), T=50 \mathrm{~K}]$ panels, classical graphene is seen to progressively develop long-wavelength ripples and so does the quantum one on top of the local roughness.

The $q$-dependent character of the classical/quantum crossover in graphene requires low temperatures for longer length scales to an extent dictated by its low bending rigidity $\kappa \sim 1-2 \mathrm{eV}$ (the precise value is still debated [14). In this respect, other 2D monolayers should display their quantum-classical crossover, albeit quantitatively less dramatic, at higher temperatures, owing to their larger values of $\kappa$. For example, the $\mathrm{MoS}_{2}$ monolayer has an area density four times higher but a bending rigidity $\kappa \sim 9-10 \mathrm{eV}[28$ ] and might therefore be more suitable than graphene for observation of quantum ripples at high temperature, due to larger value of the coefficient $\sqrt{\frac{\kappa}{\rho}}$ in the flexural mode dispersion law [see Eq.(1)]. The graphene bilayer could also be of interest in this respect - bending rigidity data in literature, however, scatter considerably, from 3.35 [29] to $35 \mathrm{eV}$ [30].

The atomistic height correlations and angular fluctuations as well as the thermal crossover just predicted are directly amenable to experimental verification, particularly through elastic scattering of impinging particles or radiation. Surface normal fluctuations of several degrees were observed in freely suspended graphene sheets at room temperature by transmission electron microscopy [31. For micron-size areas such as those realizable for suspended graphene, electron scattering by microlow-energy electron diffraction/low-energy electron microscopy is already available - so far only at temperatures of $150 \mathrm{~K}$ and higher, that is, above the quantum-classical crossover 32 34]. The intrinsic part of the diffraction peak width observed in these experiments seems indeed of the order of several degrees in monolayer graphene, and shrinks as expected on cooling from 450 to $150 \mathrm{~K}$. We predict therefore that the shrinking should cease at a crossover temperature around $50 \mathrm{~K}$ to a residual zeropoint width of the order $2^{\circ}$. At the same time, the 
roughness spectral composition should shift from longer to shorter wavelengths as indicated by Fig 4 .

To summarize, PIMC simulations provide a quantitative atomistic description of the influence of quantum mechanics on the ripples of freestanding graphene. Graphene or other kinds of 2D membranes offer a unique possibility to compare and visualize quantum and classical fluctuations in a 2D condensed-matter system. Quantum fluctuations make graphene flatter at long wavelengths, in agreement with RG studies [5, 11]. In the region of $q$-vectors spanning one decade down to $q \approx 0.06$ $\AA^{-1}$, corresponding to the length scale of about $100 \AA$, we found the height-height correlation function to follow the harmonic prediction $q^{-2}$, marginal logarithmic corrections [1] being invisible on this scale. The angular distribution of normal angles exhibits a zero-pointinduced spread of several degrees predicted to persist below a crossover temperature of about $50 \mathrm{~K}$ which will be fully measurable by scattering techniques. Other $2 \mathrm{D}$ systems, such as graphene bilayer or $\mathrm{MoS}_{2}$ with larger bending rigidities and therefore with quantitatively smaller spreads, will have higher crossover temperatures. Quantum ripples should also be relevant to low-temperature graphene tribology. Finally, the flexural fluctuations and consequent local quantum roughness are likely to affect the electronic properties of graphene at low temperatures. The importance of ripples for electron-phonon coupling was demonstrated, e.g., in Ref. [35]. Further investigations in this direction stand as an interesting problem to be considered in the future.

J. H. and E.T. would like to thank A. Morgante, S. Sorella and F. Becca for stimulating discussions. R.M. was supported by the VEGA project No. 1/0904/15 and by the Slovak Research and Development Agency under Contract No. APVV-15-0496. The calculations were performed in the Computing Centre of the Slovak Academy of Sciences using the supercomputing infrastructure acquired in Projects No. ITMS 26230120002 and No. 26210120002 (Slovak infrastructure for high-performance computing) supported by the Research \& Development Operational Programme funded by the ERDF. Work in Trieste was sponsored by ERC Advanced Grant 320796 - MODPHYSFRICT.

[1] in Statistical Mechanics of Membranes and Interfaces, edited by D. R. Nelson, T. Piran, and S. Weinberg (World Scientific, Singapore, 1989).

[2] P. L. Doussal and L. Radzihovsky, Ann. Phys. (2017), doi:10.1016/j.aop.2017.08.033

[3] Nelson, D.R. and Peliti, L., J. Phys. France 48, 1085 (1987)

[4] F. Guinea, P. Le Doussal, and K. J. Wiese, Phys. Rev. B 89, 125428 (2014)

[5] B. Amorim, R. Roldán, E. Cappelluti, A. Fasolino,
F. Guinea, and M. I. Katsnelson, Phys. Rev. B 89, 224307 (2014)

[6] A. Fasolino, J. H. Los, and M. I. Katsnelson, Nat. Mater. 6, 858 (2007)

[7] J. H. Los, A. Fasolino, and M. I. Katsnelson, Phys. Rev. Lett. 116, 015901 (2016)

[8] J. H. Los, M. I. Katsnelson, O. V. Yazyev, K. V. Zakharchenko, and A. Fasolino, Phys. Rev. B 80, 121405(R) (2009)

[9] D. Wei and F. Wang, J. Chem. Phys. 141, 144701 (2014).

[10] R. Ramírez, E. Chacón, and C. P. Herrero, Phys. Rev. B 93, 235419 (2016)

[11] O. Coquand and D. Mouhanna, Phys. Rev. E 94, 032125 (2016)

[12] E. I. Kats and V. V. Lebedev, Phys. Rev. B 89, 125433 (2014)

[13] E. I. Kats and V. V. Lebedev, Phys. Rev. B 94, 079904(E) (2016)

[14] K. H. Michel, S. Costamagna, and F. M. Peeters, Phys. Rev. B 91, 134302 (2015).

[15] B. G. A. Brito, L. Cândido, G.-Q. Hai, and F. M. Peeters, Phys. Rev. B 92, 195416 (2015).

[16] C. P. Herrero and R. Ramírez, J. Chem. Phys. 145, 224701 (2016)

[17] C. P. Herrero and R. Ramírez, Phys. Chem. Chem. Phys. 19, 31898 (2017).

[18] C. P. Herrero and R. Ramírez, J. Chem. Phys. 148, $102302(2018)$

[19] J. Tersoff, Phys. Rev. B 37, 6991 (1988)

[20] L. Lindsay and D. A. Broido, Phys. Rev. B 81, 205441 (2010)

[21] J. H. Los, L. M. Ghiringhelli, E. J. Meijer, and A. Fasolino, Phys. Rev. B 72, 214102 (2005).

[22] M. H. Müser, P. Nielaba, and K. Binder, Phys. Rev. B 51, 2723 (1995)

[23] R. Martoňák, W. Paul, and K. Binder, Phys. Rev. E 57, 2425 (1998).

[24] D. M. Ceperley, Rev. Mod. Phys. 67, 279 (1995)

[25] C. P. Herrero and R. Ramírez, J. Phys.: Condens. Matter 26, 233201 (2014).

[26] See Supplemental Material for further details about computational methods, finite-size effects, Trotter convergence, crossover wavelength and histogram of normal angles.

[27] B. Amorim, A. Cortijo, F. de Juan, A. Grushin, F. Guinea, A. Gutierrez-Rubio, H. Ochoa, V. Parente, R. Roldan, P. San-Jose, J. Schiefele, M. Sturla, and M. Vozmediano, Phys. Rep. 617, 154 (2016)

[28] K. Lai, W.-B. Zhang, F. Zhou, F. Zeng, and B.-Y. Tang, J. Phys. D 49, 185301 (2016).

[29] X. Chen, C. Yi, and C. Ke, Appl. Phys. Lett. 106, 101907 (2015).

[30] N. Lindahl, D. Midtvedt, J. Svensson, O. A. Nerushev, N. Lindvall, A. Isacsson, and E. E. B. Campbell, Nano Lett. 12, 3526 (2012).

[31] J. C Meyer, A. Geim, M. Katsnelson, K. S Novoselov, T. Booth, and S. Roth, Nature (London), 446, 60 (2007)

[32] K. R. Knox, S. Wang, A. Morgante, D. Cvetko, A. Locatelli, T. O. Mentes, M. A. Niño, P. Kim, and R. M. Osgood, Phys. Rev. B 78, 201408 (2008).

[33] A. Locatelli, K. R. Knox, D. Cvetko, T. O. Mentes, M. A. Nio, S. Wang, M. B. Yilmaz, P. Kim, R. M. Osgood, and A. Morgante, ACS Nano 4, 4879 (2010) 
[34] K. R. Knox, A. Locatelli, M. B. Yilmaz, D. Cvetko, T. O. Mentes, M. A. Niño, P. Kim, A. Morgante, and R. M. Osgood, Phys. Rev. B 84, 115401 (2011)

[35] A. Laitinen, M. Oksanen, A. Fay, D. Cox, M. Tomi,
P. Virtanen, and P. J. Hakonen, Nano Lett. 14, 3009 (2014) 